\title{
Sciendo
}

ACTA UNIVERSITATIS CIBINIENSIS - TECHNICAL SERIES

Vol. 712019

\section{DETERMINATION OF THE NUMBER OF OBJECTS THROUGH WEIGHT AND IMAGE PROCESSING}

\author{
Laura-Diana Iamandi-Gido \\ “Lucian Blaga”University Sibiu, Romania, laura.iamandi@ulbsibiu.ro \\ Mihai Neghină \\ “Lucian Blaga”University Sibiu, Romania, mihai.neghina@ulbsibiu.ro
}

\begin{abstract}
Estimating the number of identical objects is a fundamental recognition task that requires exhaustive human effort. Automating this task in a warehouse would reduce the human load significantly. In this paper, we propose a method to automatically detect the number of objects, requiring only low-tech hardware and low-processing software, in a manner that provides optimal performance for the needed tasks. This method combines the physical weight of objects and simple image processing that do not require complicated operations, such as the detection of objects and their locations or the extraction of object figures. The experimental results demonstrate the increased effectiveness of the proposed method compared to methods based on weight alone or on image processing alone.
\end{abstract} $\operatorname{logic}$

Key words: detecting number of objects, weight processing, image processing, morphological operations, fuzzy

\section{Introduction}

As technology evolves, an increasing number of products are becoming smaller than their previous models. Correspondingly, components used to manufacture these products are also becoming smaller and smaller. In effect, the smaller and lighter the object is, the more significant the errors in measuring the size or weight of the object become. Processing these components after manufacturing can be challenging, especially when it comes to packaging or keeping inventory.

Weighing is one of the most popular methods in the industry for estimating the number of objects, but variances between small parts and the oscillations from the environment can lead to significant deviations. In packaging, such variance can lead to shortage or surplus of parts, with no actual indications of the level of inaccuracy [1]. Most relevant developments for the industry regarding the inference of the number of (accurately) weighed objects are usually included in patents, some even spanning more than 100 years $[2,3,4,5]$.

Image processing is another important approach to object detection, identification and quantity estimation, as the advancing of sensors quality and processing power provide excellent tools. The problem of number estimation of objects in an image is addressed through a multitude of methods that process different features of the objects. Edge detection and Hough circle transform has been used by Baygin et al. [6] for counting non-overlapping eggs and soda bottles on a conveyor. Bera [7] proposed a method for counting partially occluded round objects, where boundaries of the object patches have been extracted using morphological operations and the exact coordinates of their boundaries has been computed with the help of Euclidian geometry. Gu et al. [8] attempted the detection of circular objects by adaptive filtering and combining low-level feature descriptors and a linear SVM.

Pattern matching as a method for identifying and locating specific objects, regardless the background or other objects present in the image, has also been a popular choice in literature $[9,10]$. Neural networks have also been successfully applied to object detection when there are different types of objects to be counted separately, or when the objects of interest must be identified amidst several other objects that are not to be considered. But the estimation of the number of objects can be done without locating or identifying the objects as well. Zhao and $\mathrm{Li}$ [11] used a vibrating mechanism to disperse grains and seeds and compute their area from images after applying morphological operations.

The proposed method aims to combine imprecise measurements, such as weight, area and perimeter, without identifying the objects and their locations. Combining data obtained through such different measurements with a fuzzy logic decision making process can help in coming to the correct estimation of the number of objects. 
The remainder of the paper is divided as follows: section II describes the proposed method for estimating the number of identical objects, section III describes the experimental setup and presents the results, while the final section discusses the conclusions and perspectives.

\section{Proposed Method}

Both the image processing methods and the weighing methods, when used by themselves, have specific issues when applied to identifying the number of relatively small objects. The proposed method therefore tries to improve the reliability of the results by combining the data obtained through images and weights. The flowchart in Fig.1 shows the two branches of processing: the database algorithm and the main algorithm for estimating the number of objects.

For each type of object, generic data needs to be stored in a database, including the dimensions of one piece and the mean reflectance. The acquisition of this information has to be done only once, since the data is valid for all components of the same type. The operations necessary are similar to the operations in the image processing branch of the main algorithm, except for the computation of the reflection rate, which will be discussed separately after the main algorithm.

In the main algorithm, the image processing branch generates two estimates for the number of objects, based on the total area and perimeter of the region of interest, while the weight acquisition branch generates one estimate, all three estimates being fed into a fuzzy logic decision block for the final result.

The following sub-sections describe in more detail the processing steps of the flowchart diagram in Fig. 1.

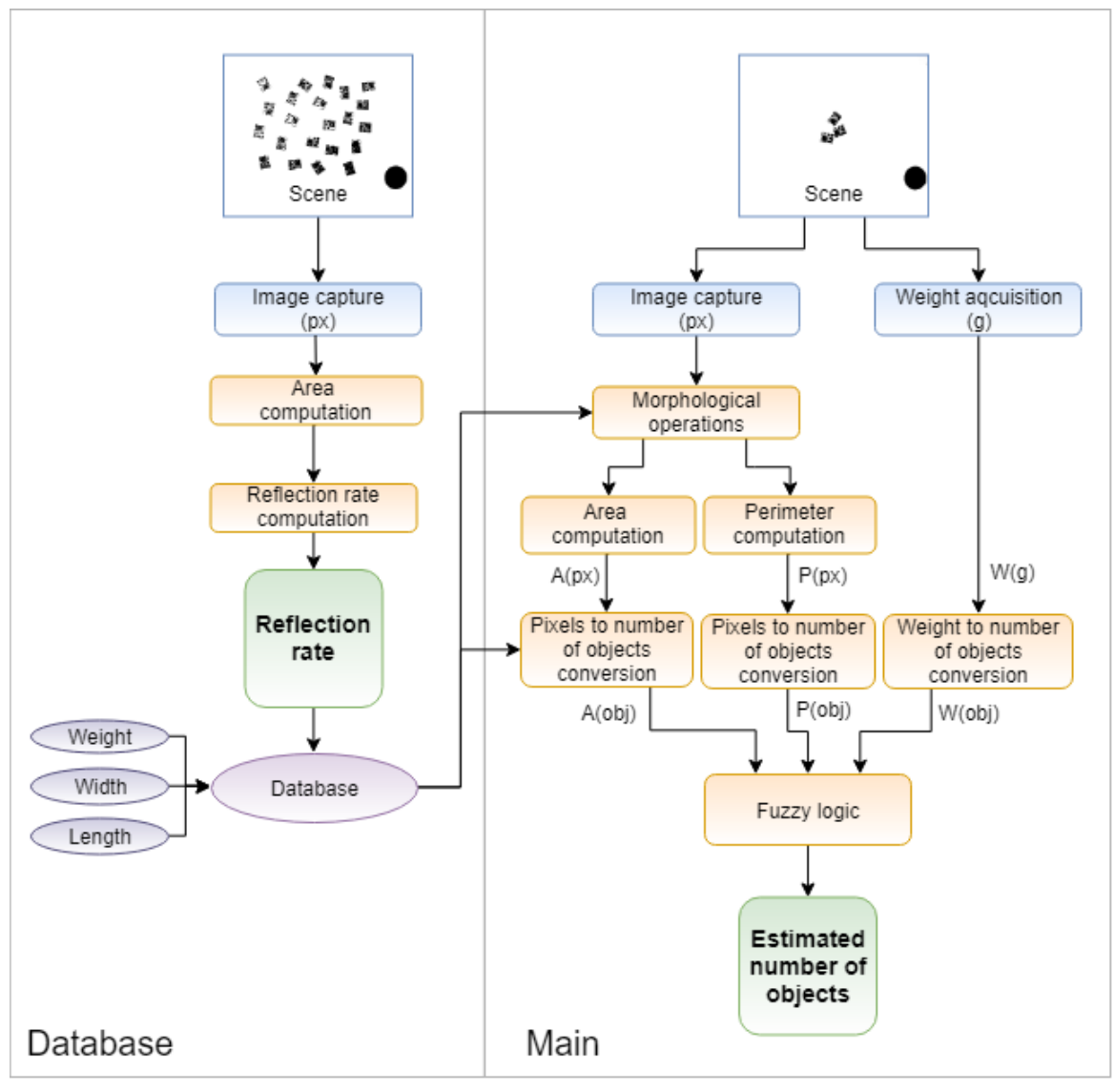

Figure 1 Flowchart of the proposed objects number estimation algorithm

A) Main algorithm

1) Image acquisition and binarization. The image is captured by a camera, at a variable distance from the objects. Considering the types of objects in the experiment, mainly electronic modules, one of the most important impediments in acquiring a correct image is light reflection. There are several techniques that deal with reflection, such as polarized light and filters [12] or spectral composition [13]. One of the cheapest and accessible method is using differently positioned sources of light, while the camera captures one image for each. The two images are then overlapped, providing a better image. The image acquisition returns a high-contrast grayscale image, on which Otsu's method of binarization [14] is applied. 
2) Morphological operations. The morphological closing operation is applied to binary images. Dilation, performed first, tends to close gaps and holes still present in the object because of light reflection, while erosion shrinks the objects back to aproximately the original size, without allowing the holes to reappear. For multiple objects, closer to each other than the structuring element, the side-effect is that the gap between them will be filled, increasing the overall area and, generally, decreasing the perimeter. The structuring element has been chosen as a disk with variable radius $\mathrm{R}$, depending on the reflection rate of the object type $\mathrm{r}$ (determined in section IIB1) and the object width 1 (in pixels) as in eq. (1).

$$
R=(r * l) / 3
$$

3) Area computation. Area is determined as the number of black pixels. Knowing the ratio between pixels and millimeters, the number of objects can be estimated by dividing the resulted area with the one from database.

4) Perimeter computation. Image filtering is done using sparse kernel matrix convolution. Matrix size has been chosen empirically, so as to best eliminate pins and leads of the electronic components. The result of this operation is a perimeter whose thickness is proportional to the matrix size. The equivalent 1 pixel thick perimeter estimation can be then computed by multiplication with a correction coefficient.

5) Pixels to number of objects conversion. Since the camera doesn't have a fixed position and the distance from it to the tray may vary, a reference mark of known dimensions is placed on the tray. Using this mark, we are able to compute the ratio between the metric dimension (millimeters, in this case) and pixels. This operation is essential, since all the dimensions stored in the product database are metric. Knowing this ratio, the estimated number of objects is computed taking into account the reference area and perimeter of the object stored in the database.

6) Weight acquisition. The weight is acquired using a $1 \mathrm{~kg}$ load cell and an analog to digital convertor. For better results provided by the weighing system, several readings are necessary, in order to use their average and to eliminate some of the effect of oscillations coming from the work environment.

\section{7) Weight to number of objects conversion}

The estimated number of objects is computed taking into account the reference weight of the object, which had been previously stored in the database.

8) Decision making (fuzzy logic). There are a few situations where the area can be erroneously computed. Area becomes smaller, as the reflection rate increases, and conversely becomes larger, as the objects are closer and the gaps between them are filled by the morphological operations. As opposed to the area, higher the reflection rate, bigger the perimeter and closer the objects, smaller the perimeter. Morphological operations and filters may improve the result, but a certain tolerance still exists. The weighing scale is also imprecise, having a tolerance of $20 \%$.

These 3 estimations of the number of objects - coming from the area, perimeter and weight - represent the input variables of the fuzzy logic decision making. Their fuzzy memberships are represented in Fig. 2. The area membership functions are more extended to the right because the image processing operations have a higher chance of including additional pixels in-between close objects, whereas the number of missing pixels is minimized through the use of dual lighting. The perimeter membership functions are more relaxed than the membership functions for the area because the perimeter value is the least reliable of the three parameters for estimating alone the number of objects. The weight membership function is balanced since there is no indication that the scale should have any bias. For all membership function families, larger numbers of objects are characterized by wider functions.

All aggregation rules are of the same type: if the number of objects estimated by area is $k$ and by perimeter is $k$ and by weight is $k$ then the number of estimated objects is $k$.

When the fuzzy aggregation function based on rules creates a plateau at the maximum value, the defuzzification method is Middle of Maximum (mom), rounded to the closest integer. In the case that no rule has fired, the output class is "fail", which means that the value of at least one of the three estimates is incompatible with the other two estimates. Failed experiments are considered inconclusive rather than incorrect, since the experiment can be rerun. 


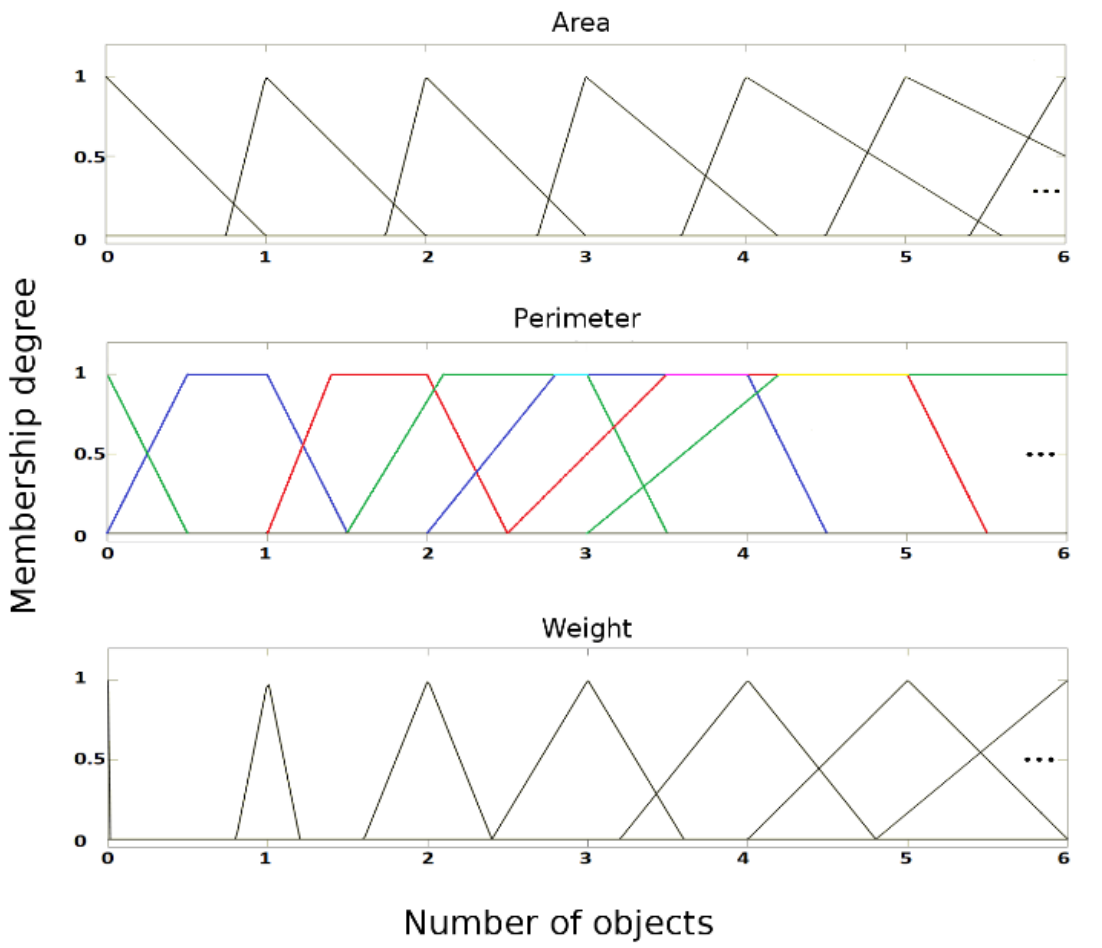

Figure 2 Fuzzy memberships plot

Database

The database contains two types of data for each object type:

- Manufacturer-provided data: length, width, perimeter and weight for one object, (used in sections IIA5 and IIA7)

- Computed data: the light reflection rate for one object (used in section IIA2).

If the area or the perimeter are not provided by the manufacturer, irregularly shaped objects may be time consuming to measure accurately. However, this needs to be done only once for each type of object and the operation can coincide with de reflection rate acquisition.

Reflection rate computation. Reflection rate $r$ is a measure of average undetected area due to reflection, computed with the database area $A_{D B}$ and the equivalent acquired area $A_{a}$ for one object, according to equation 2.

$$
r=\frac{A_{D B}-\mathrm{A}_{a}}{A_{D B}}
$$

As it can be seen in Fig. 4, the light reflection is not uniform over the tray surface, so the area $A_{a}$ must be acquired by uniformly placing a known quantity of objects on the tray.

\section{Results}

A) Experimental setup

The mechanical ensemble is represented in Fig. 3. Each element is described in detail in the following sections.

1) Height adjustable camera. A Logitech $\mathrm{C} 310$ webcam has been used to capture pre-processed images. The following parameters have been adjusted in order to get a white-background high-contrast grayscale image: exposure, gain, brightness, contrast, color intensity, white balance. For faster computation, the image is downsized to $640 \times 480$ pixels. 


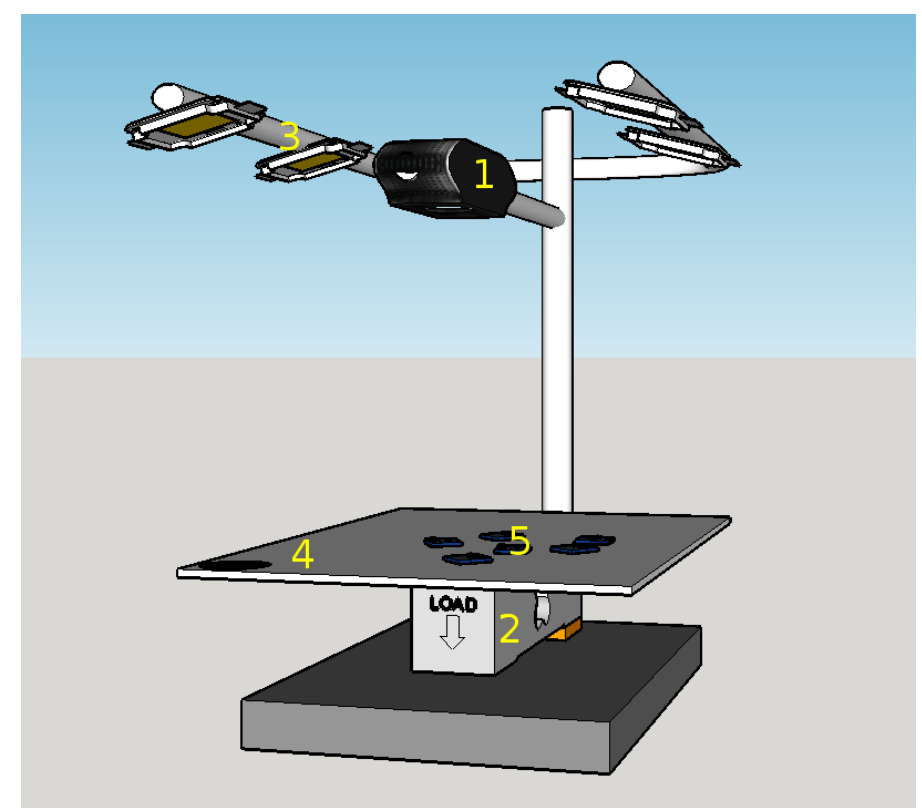

Figure 3 Physical setup. 1-height adjustable camera. 2-load cell and ADC 3-illumination sources. 4white tray with reference mark. 5-objects

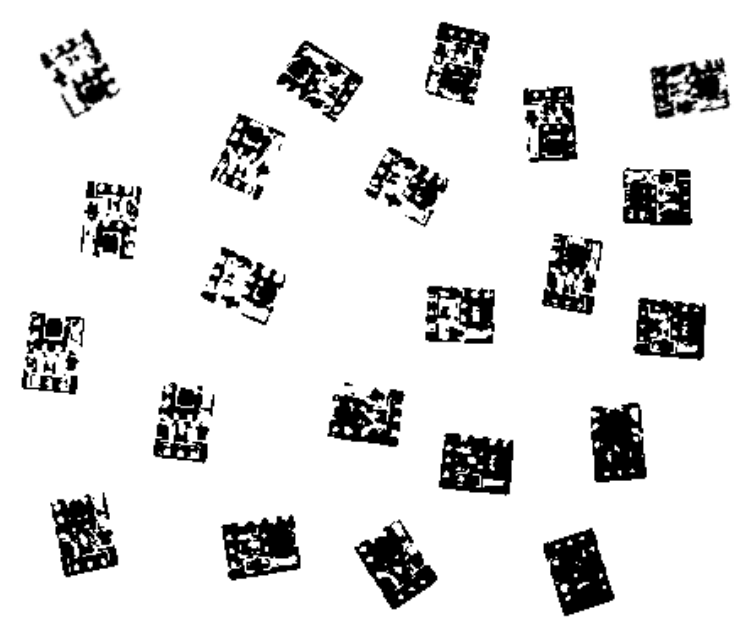

Figure 4 Example of pre-processed captured image

2)Load cell and ADC. HX711 is a 24-bit ADC, programmed with a gain of 128, corresponding to a fullscale differential input voltage of $\pm 20 \mathrm{mV}$ when a $5 \mathrm{~V}$ supply is connected. The scale is designed for a maximum weight of $1 \mathrm{~kg}$ and the output value has a precision of 2 decimals.

Due to time constraints, an average result of 10 readings, equivalent to $1300 \mathrm{~ms}$ of computation, is chosen for this experiment (Fig. 5).

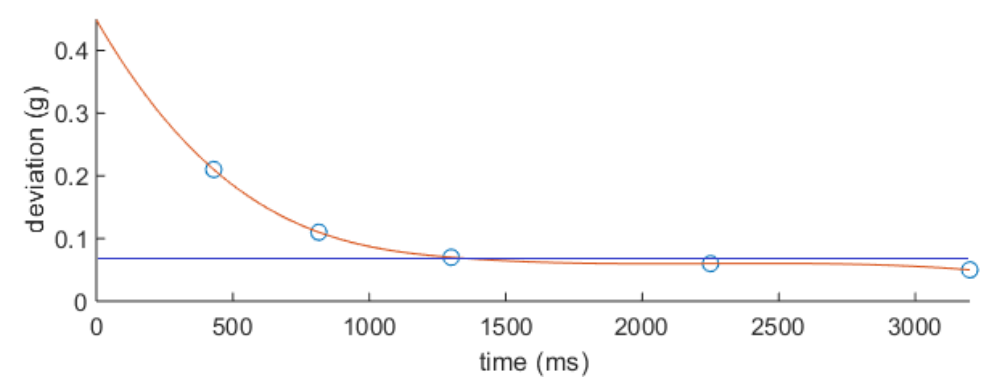

Figure 5 Scale deviation vs computation time 
Load cell's accuracy class provided by its datasheet is $0.05 \%$. However, in tests, due to oscillations in the work environment, the deviation proved to be up to $20 \%$ for smaller weights (Fig. 6).

3) Illumination. The illumination is done with 2 differently positioned sources of light $(4 \times 48 \mathrm{COB}$ LED, 4w, 5500k, 720lm) that are activated alternatively, resulting a significant reduction in light reflection

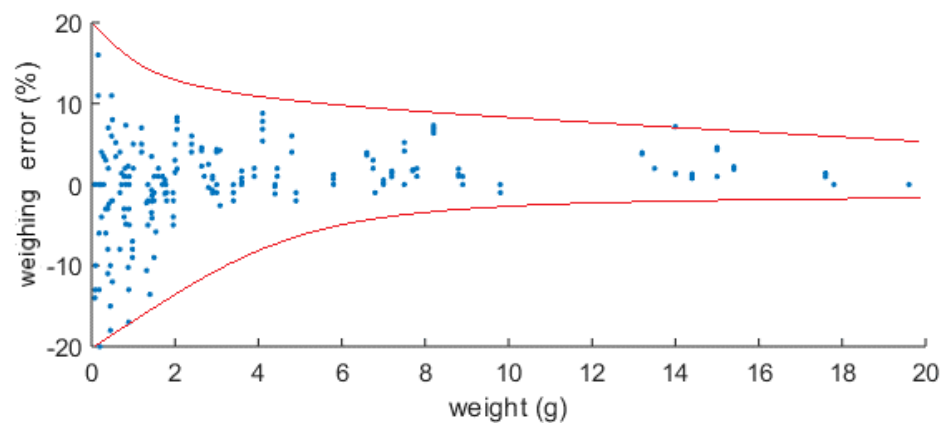

Figure 6 Weighing error for different weights

4) Reference mark. A $25 \mathrm{~mm}$ radius reference mark has been arbitrary chosen, in order to measure the objects, regardless the distance between camera and tray. It is round shaped, placed on bottom's right corner and it is measured by cropping the corner at the maximum size the mark can have, in the given setup.

5) Tested objects. 18 different object types were tested, mainly electronic components and modules: PCBs, connectors, ceramic capacitors. For each type, the algorithm was tested with 1, 2, 5, 10 and 20 identical non-overlapping, touching items, summing up 245 cases. The objects are rectangular or round shaped, with an area between 1.61 and $558.15 \mathrm{~mm} 2$ and weight between 0.08 and $1.54 \mathrm{~g}$. Their volume is close to 0 , so it is neglected in experiments.

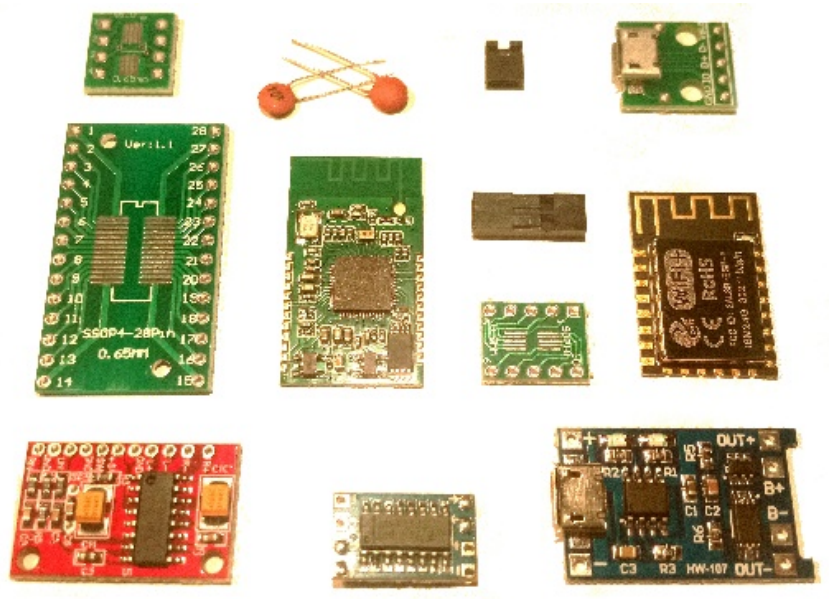

Figure 7 Example of objects used in tests

B) Performance Evaluation

In order to evaluate the performances of the proposed method, the following parameters have been chosen:

$\mathrm{CE}=$ correct estimations - the number of experiments where the correct amount of objects has been estimated

$\mathrm{TE}=$ total estimations - the total number of experiments

$\mathrm{IE}=$ incorrect estimations - he number of experiments where the incorrect amount of objects has been estimated

$\mathrm{FE}=$ failed estimations - the number of failed experiments, for which the fuzzy logic rules cannot be aggregated into an estimate

Correct detection rate [\%]:

$$
C D R=C E / T E * 100
$$

Incorrect detection rate $[\%]$ :

$$
I D R=I E / T E * 100
$$

Fail detection rate $[\%]$

$$
F D R=F E / T E * 100
$$




\section{C) Experimental results}

The positioning of the pieces on the tray has been chosen so that the test cases are as varied as possible, including pieces far apart from each other (best scenario) up to pieces clustered together (most challenging scenario). For about a third of tests having more than two pieces, the objects were randomly arranged on the tray, simulating a real situation where, for example, an automatic feeder or a robotic arm places them.

Table I represents the performance of the proposed combined method compared to each branch alone. The proposed method managed to increase the estimation accuracy from $86.99 \%$ (weight), $73.57 \%$ (area) and $59.53 \%$ (perimeter) up to $90.61 \%$, by combining the results of the 3 measurements. A significant advantage of this method is that it provides "fail" result, if no fuzzy rule is fired. This may happen in cases where the objects are too close to each other or the reflection rate is too big. In this case, the objects can be rearranged for a new measurement, so that the correct detection rate may go up to $93.47 \%$.

TABLE I. Performances of the proposed method

\begin{tabular}{|l|c|c|c|c|}
\cline { 2 - 5 } \multicolumn{1}{c|}{} & Combined method & Only weight & Only area & Only perimeter \\
\hline CE & 222 & 213 & 2 & 146 \\
IE & 16 & 32 & 243 & 99 \\
FE & 7 & 0 & 0 & 0 \\
TE & 245 & 245 & 245 & 245 \\
CDR [\%] & $90.61 \%$ & $86.99 \%$ & $73.57 \%$ & $59.53 \%$ \\
& & $-3.62 \%$ & $-17.04 \%$ & $-31.08 \%$ \\
IDR [\%] & $6.53 \%$ & $13.01 \%$ & $26.43 \%$ & $40.47 \%$ \\
FDR [\%] & $2.86 \%$ & $0 \%$ & $0 \%$ & $0 \%$ \\
CDR+FDR [\%] & $93.47 \%$ & $86.99 \%$ & $73.57 \%$ & $59.53 \%$ \\
& & $-6.48 \%$ & $-19.90 \%$ & $-33.94 \%$ \\
\hline
\end{tabular}

The confusion matrix of the proposed method presented in table II reveals that the estimation error is 0 for $1-5$ objects and grows up to $10 \%$ for 20 objects. The fail detection rate suggests how many measurements should be retaken and it increases with the number of objects. As a consequence of the considered membership functions, it can be also observed that the estimation error is always positive. For other sets of objects, the fuzzy membership functions and decisions can be adapted, based on specific requirements.

TABLE II. Confusion matrix for the proposed method

TABLE III. Confusion matrix for weight only
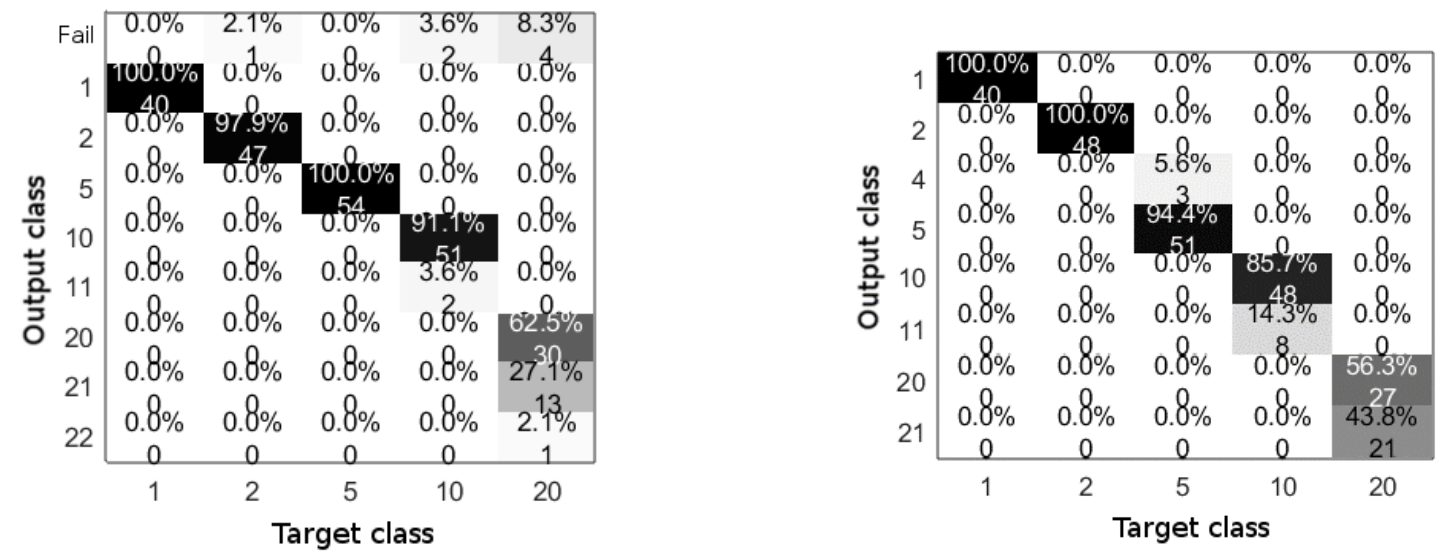

For comparison, table III presents the confusion matrix for weighing only. Without the "fail" case or other controls over the results, the estimation error is both positive and negative.

\section{Conclusions / Discussions / Perspectives}

The proposed method managed to increase the number estimation accuracy from $86.99 \%$ (weight) to $90.61 \%$ and potentially up to $93.47 \%$, by combining the results of the 3 measurements.

A significant advantage of this method is that it provides "fail" result, if no fuzzy rule is fired. This may happen in cases where the objects are too close to each other or the reflection rate is too big. An easy solution may be the rearrangement of objects for a new reading (case in which the fail detection rate suggests how many measurements should be retaken) or taking into consideration the result of the most reliable measurement, the scale in this case, assuming its error.

The fuzzy membership functions and decisions can be also adapted, based on specific requirements: for example, if it's very important to correctly estimate the number of objects, more narrow ranges of decision are preferable, even if that means more failed cases. 
Having control over the fuzzy membership functions allows the shift towards negative or positive side the estimation error, based on requirements. For example, when packing electronic components for a doit-yourself kit, it is much more preferable to pack an extra component than to deliver an incomplete kit. Conversely, when making an inventory, it's better to estimate less than the real quantity of the stock. For the experiments presented in this paper, a positive error has been preferred.

This method is ideal for many relatively small objects, that weigh less than the scale accuracy, so that the image processing contribute to the result. It is also suitable for through-hole electronic components, such as capacitors or resistors, seeing that the filtering operation eliminates the pins and leads and deals only with their package (body). The objects can also touch each other. For further development, a vibrating mechanism that disperse the objects automatically could be integrated.

The reduced set of operations allows fast processing and adaptation for wide range of objects, while the low-tech, basic hardware is sufficient and provides easy and accessible setup.

As presented, the method is limited to relatively flat objects that are not (significantly) overlaid on the tray, since volume creates shadows that would distort the image processing estimations.

\section{Acknowledgment}

This work is supported through the DiFiCIL project (contract no. 69/08.09.2016, ID P_37_771) co-founded by ERDF through the Competitiveness Operational Programme 2014-2020.

\section{References}

[1] "Solving challenge of part weight practice by count," DATA Detection Technologies LTD, Israel, 2019. [Online].Available: https://data-technologies.com/wp-content/uploads/2019/01/Solving-thechallenges-of-part-weight-practice-by-count.pdf

[2] W. Sanderson, "Weighing and counting machine", Patent US659830A, issued October 16, 1900

[3] R. L. Marcheso, B. Lukaschewsky, " Method and apparatus for calculating a piece count by weighing calculations", Patent US3552511A, issued January 5, 1971.

[4] C. Liang, "Warehouse management system for automatically weighing and counting individual parts", Patent US8606660B2, issued December 10, 2013.

[5] L. Youjun, "Number rechecking device for weighing and counting production line", Patent CN204368557U, issued June 3, 2015

[6] M. Baygin, M. Karakose, A. Sarimaden, E. Akin, "An Image Processing based Object Counting Approach for Machine Vision Application”, ICAIE, 2017.

[7] S. Bera, "Partially Occluded Object Detection and Counting", Proc. Of Third International Conference on Computer, Communication, Control and Information Technology (C3IT), India, 2015

[8] Y. Gu, L. Li, F. Fang, M. Rice, J. Ng, W. Xiong. J.H. Lim, "An Adaptive Fitting Approach for the Visual Detection and Counting of Small Circular Objects in Manufacturing Applications", 26th IEEE International Conference on Image Processing (ICIP), Taiwan, 2019

[9] J. K. Dharpure, M. B. Potdar, M. Pandya, "Counting objects using convolution based pattern matching technique”, International Journal of Applied Information Systems, vol. 5 (8), 2013

[10] C. Nudol, "Automatic jewel counting using template matching", IEEE International Symposium on Communications and Information Technology, Japan, 2004

[11] P. Zhao, Y. Li, "Grain Counting Method Based On Image Processing”, International Conference on Information Engineering and Computer Science, China, 2009

[12] H. Fujikake, K. Takizawa, T. Aida, H. Kikuchi,T. Fujii, M. Kawakita, "Electrically-Controllable Liquid Crystal Polarizing Filter for Eliminating Reflected Light" Optical Review, 5(2), 93-98, 1998

[13] H.-C. Lee, E. J. Breneman, C.P. Schulte, "Modeling light reflection for computer color vision”, IEEE Transactions on Pattern Analysis and Machine Intelligence, 12(4), 402-409, 1990

[14] N. Otsu, "A Threshold Selection Method from Gray-Level Histograms", IEEE Transactions on Systems, Man, and Cybernetics, 9(1), 62-66, 1979 